\title{
Research on Electromagnetic Scattering Characteristics and Radar Cross Section Reduction of Vertical tail
}

\author{
Huang Haoran ${ }^{1, a}$, Wang Hongwei ${ }^{1, a}$, Sun peng ${ }^{1, b^{*}}, C^{2}$ i $\mathrm{yu}^{2, \mathrm{a}}$, \\ Jiang Zhaochun ${ }^{3, a}$, Liang shicong ${ }^{4, a}$ \\ ${ }^{1}$ Department of Aircraft and Dynamics, The Aviation University of Air Force, Changchun \\ 130022,China \\ ${ }^{2}$ Equipment department of Shenyang Air Force, Shenyang, 110000,China \\ ${ }^{3}$ Representative Office in Qingdao Area of Air force, Qingdao 266000,China \\ ${ }^{4}$ Equipment department of NO.94836 Troops, Nancang, 330201,China) \\ awhw1001@126.com, brocy527@163.com
}

Key words: Stealth technology;Vertical tail;Radar cross section;Method of moment

Abstract: Method of Moment is used to calculate vertical tail's radar cross section(RCS). The impact of leading-edge sweep angle, span and inclination on vertical tail's RCS is analyzed and then vertical tail's RCS curves and function expressions with leading-edge sweep angle,span and inclination are fitted by the calculation results. The results show that by optimizing the vertical tail leading-edge sweep angle, span, inclination and other geometrical parameters, can improve the stealth performance of the vertical tail. To optimize the design of the vertical tail of the stealth provide the technical foundation.

\section{Introduction}

According to aircraft radar cross source analysis, the aircraft is a very complex targets by multiple components. Under the radar illumination, each component will produce scattered waves, some parts may also produce different scattering mechanisms scattering sources, the formation of multiple scattering sources. As the aircraft RCS is a comprehensive source of each scattering results, it is easy to understand and reduce the RCS values for each scattering source is likely to make the RCS reduction in the value of the whole. For this reason, the design of the aircraft, should try to reduce the value of the relevant parts of the RCS. The aircraft's tail as an important radar cross source of aircraft, reducing its RCS to improve the stealth aircraft plays an important role. Therefore, it is necessary to study the characteristics of the radar cross of the tail and look for ways to reduce its RCS.

Firstly, the establishment of the tail of the three-dimensional model, model RCS calculated using the method of moments, focuses on studying the impact of leading-edge sweep angle, span and inclination on vertical tail's RCS and then vertical tail's RCS curves and function expressions with leading-edge sweep angle,span and inclination are fitted by the calculation results.

\section{MoM principle of the method}

Integral equation commonly used method of moments (method of moment, MoM) to solve. The mathematical nature is a method for solving linear equations. 
The basic principle of the method of moments is to use a number of discrete sub-domains to represent the entire contiguous area. In each sub-domain,unknown function with a base function with unknown coefficients to represent, and therefore issue an unlimited number of degrees of freedom was transformed into a finite number of degrees of freedom of the problem, then use the point matching, line matching method, Galerkin gold method or other methods for inspection, get a set of algebraic equations, and finally obtain the numerical solution by solving the matrix equation.

Moment method for solving integral equations include the following steps: divide into discrete regions or target; select the appropriate basis functions and test functions; fill the impedance matrix; solve the matrix equation.

For example, the operator equation:

$$
L\{f(x)\}=g(x)
$$

$L$-linear operator, $f(x)$ - the unknown of unknown function, $g(x)$ - known function. To solve this equation, you need to first launched as a series of unknown function known function (basis functions) superimposed forms, namely:

$$
f(x) \approx \sum_{n=1}^{N} a_{n} b_{n}(x)
$$

Where: $a_{n}$-the unknown coefficients of the basis functions;

$b_{n}$ - the $n$th basis functions.

\section{Vertical tail radar scattering analysis}

Vertical tail geometrical parameters which affect RCS are: leading-edge sweep angle $\chi$, $\operatorname{span} b_{c}$ and inclination $\theta$. Here, we use Feko software to simulate Vertical tail electromagnetic simulation models which have different above the geometric parameters, find its RCS.

Firstly, establish vertical tail of the three-dimensional model, model specific parameters: airfoil NACA0009, the root of the chord length $c_{c}=3.1 \mathrm{~m}$,span $b_{c}=3.7 \mathrm{~m}$, the trailing-edge sweep angle $20^{\circ}$, the leading edge sweep angle change from $30^{\circ}$ to $47.5^{\circ}$. Using the method of moments calculated RCS curve shown in Figure1.

The RCS average (the star in Figure 2) within the pitch angle range $-20^{\circ} \sim 20^{\circ}$ using the least squares fitting method to obtain the curve shown in Figure2.

As can be seen from Figure 2 , in the range $\pm 20^{\circ}$ of the pitch angle, as the leading edge sweep angle increases, the value of RCS decrease. According to the results, the relationship between the leading-edge sweep angle and the value of RCS be fitted curves in the graph, the expression is as follows:

$$
S=-0.0023 \chi^{2}+0.0114 \chi-26.3780
$$




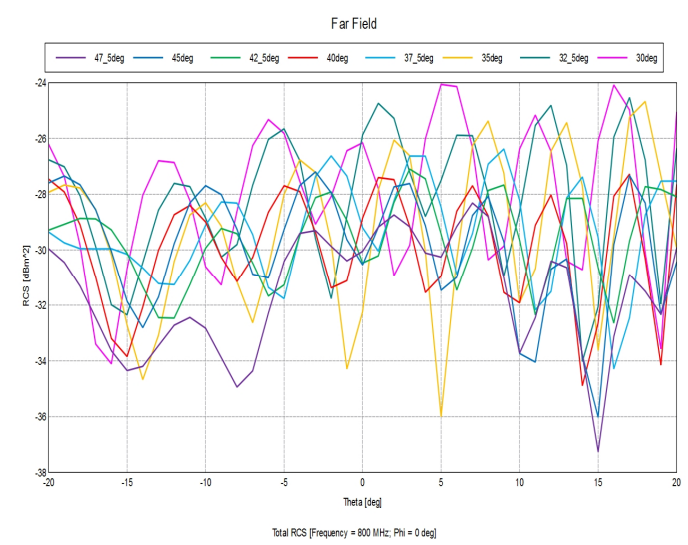

Figure1 RCS curve with the leading-edge sweep angle changes

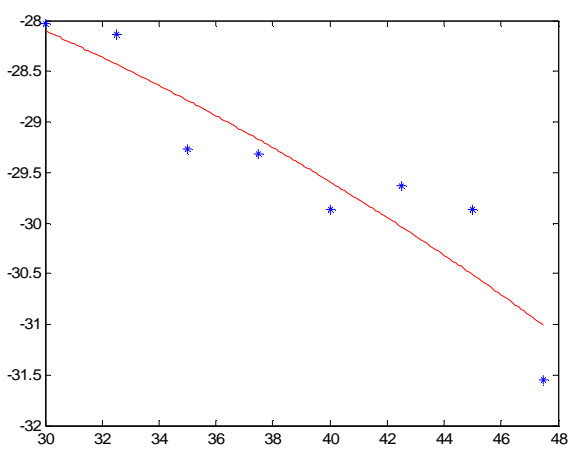

Figure2 The impact of leading-edge sweep angle on RCS

By Equation (3) shows that, with the leading edge sweep angle increases, the average value of RCS is reduced to speed up. This suggests that further increases when the leading edge sweep angle, RCS reduction benefits will be even greater.But we must point out that this would have paid greater aerodynamic performance cost.

Keep the vertical tail model airfoil and root chord invariant, leading-edge and trailing-edge sweep sweep and were fixed at $40^{\circ}$ and $20^{\circ}$. Span increases from $3.4 \mathrm{~m}$ to $4.1 \mathrm{~m}$,calculate RCS value curve of vertical tail model, as shown in Figure3. And vertical tail model fitting curves is plotted in Figure 4 in the pitch angle range of $\operatorname{RCS}$ averages $-20^{\circ} \sim 20^{\circ}$ within.

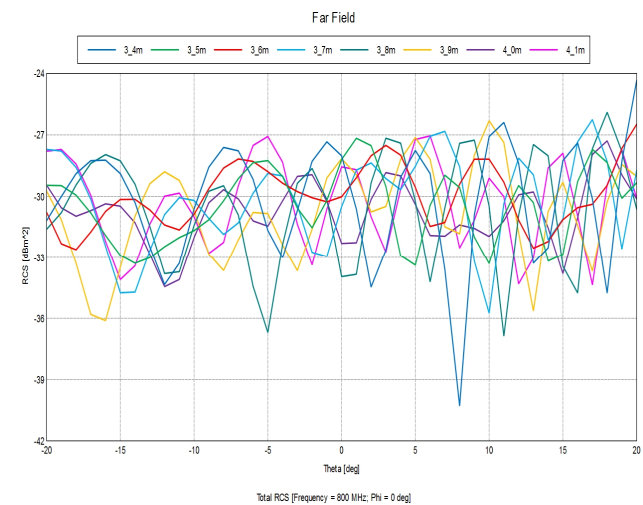

Figure 3 RCS curve with the span changes

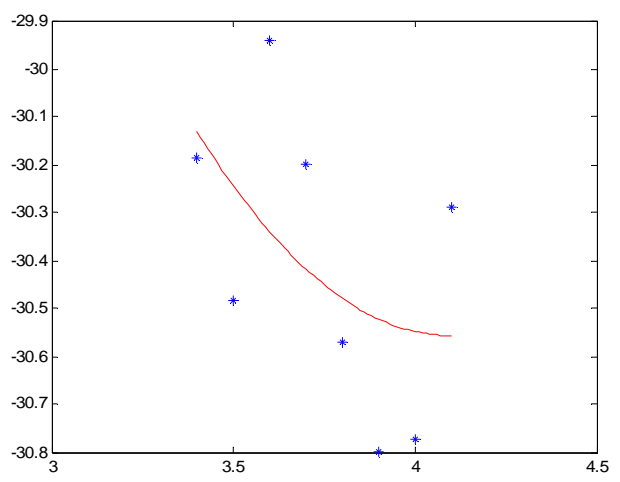

Figure 4 Impact of span on the RCS

As can be seen from Figure4, the average value of RCS (the star symbol in Figure4) with the distribution of the span development is unstable. Fit the relationship between the value RCS and the span to quadratic curve approximately,showed as Figure 4, the RCS value can be obtained with the development span increases, the expression of the curve as following :

$$
S=0.8757 b_{c}^{2}-7.1768 b_{c}-15.8524
$$

By equation (7) shows that, for this airfoil when span of less than $3.7 \mathrm{~m}$, the average value of RCS decreases approximately linearly with the span; when more than $3.7 \mathrm{~m}$, showed a non-linear relationship between the two, RCS decreases slower. This suggests that, when the span is greater than a value, further increasing the span, RCS reduction effect is reduced. then the value for span, should weigh the other requirements of pneumatic and strength. 


\section{summary}

In this paper, using the moment method to calculate the tail of RCS, draw the relationgship between the tail RCS and the leading-edge sweep angle, the span,the inclination: increase the value of $\chi$, RCS decreases; $b_{c}$ increases, RCS increases ; $\theta$ increases, RCS decreases. Thus obtained in the case of the tail meets the aerodynamic characteristics, strength requirements and other conditions, by appropriate increase, decrease, increase to optimize the fin geometry parameters to achieve it RCS minimum. In addition, the tail can be reduced through the use of RCS absorbing materials.

\section{References}

[1]Zhang Kao, Ma Dongli. military aircraft survivability and stealth design Beijing: National Defense Industry Press, 2002.

[2]Li Yi. radar electromagnetic scattering calculation and experimental research stealthy targets. Changsha National University of Defense Technology, 2007.

[3]Sang Jianhua. Aircraft stealth technology. Aviation Industry Press, 2013.

[4]Jiao Zihan, Zhang Binqian, Shen Dong. Airfoil geometric parameters on stealth characteristics. Mechanical Science and Technology, 2012.12.

[5]Li Qipeng, Wang Heping, Sun Zhen, Fu Wei. Scattering of canard Research and RCS reduction. Aeronautical Computing Technique, 2010.

[7]Weinmann F.Ray Tracing With PO / PTD for RCS Modeling of Large Complex Objects [J] .Antennas and Propagation'IEEE'2006 (6): 1797-1806. 